\title{
Europäische Geldpolitik: fehlkonzipiert, irregeleitet und lernresistent
}

Kritik an der unabhängigen Europäischen Zentralbank gilt in Deutschland mit seiner durch die Bundesbank über Jahrzehnte geprägten „Stabilitätskultur“ als verpönt. Doch war ihre Politik wirklich so erfolgreich wie von ihren Repräsentanten gerne behauptet wird? Der folgende Beitrag hinterfragt kritisch die Rolle und die Politik der Europäischen Zentralbank (EZB) innerhalb der Europäischen Währungsunion (EWU). Die grundsätzliche Konzeption und die praktische Durchführung europäischer Geldpolitik seit dem Vertrag von Maastricht können dieser Analyse zufolge nicht überzeugen.

\section{Einleitung}

In ihrer Selbstdarstellung hat die Geldpolitik der Europäischen Zentralbank (EZB) mit einer durchschnittlichen Jahresinflationsrate von 1,97\% seit Beginn der europäischen Wirtschafts- und Währungsunion (WWU) eine wahre Meisterleistung vollbracht. Die heutige Krise der WWU sei allein auf das stabilitätswidrige wirtschaftspolitische Verhalten der Länder mit exzessiven Haushaltsdefiziten zurückzuführen. Die Wiederherstellung von Stabilität erfordere das Einschwenken dieser Länder auf den durch Deutschland vorgegebenen Pfad der Tugend (Trichet 2011).

In kritischer Absicht arbeite ich zunächst die hervorgehobene Position der EZB im Maastrichter Regime der WWU und seine generelle Fehlkonstruktion heraus (Abschnitt 2). Sodann wird aufgezeigt, dass der vermeintlich stabilitätsorientierte Kurs der EZB durch schwere Politikfehler gekennzeichnet ist, die zu gravierenden Fehlentwicklungen maßgeblich beitrugen, einschließlich jener Ungleichgewichte und Verwerfungen, die den Hintergrund zur heutigen Krise der WWU bildeten (Abschnitt 3). Die durch diese Krise erzwungenen Reaktionen der EZB zeigen allerdings, dass auch die EZB inzwischen zum Euro-Verlierer geworden ist, da ihr eigenes Schicksal selbst bei unantastbarer Unabhängigkeit an das Schicksal des Euro gekoppelt bleibt (Abschnitt 4). Und um den Euro und den Prozess europäischer Integration steht es nicht gut. Allerdings gerade weil das fehlkonzipierte Maastrichter Regime zu einer Pervertierung deutscher Stabilitätskultur auf europäischer Ebene führt.

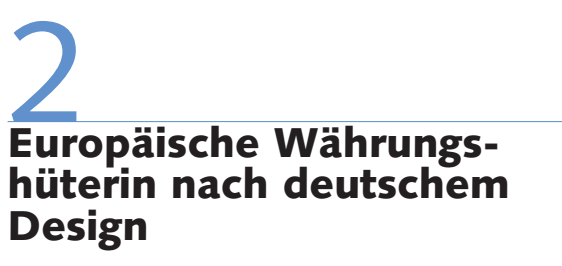

Eine Beurteilung der Politikleistung der EZB bewegt sich notwendigerweise vor dem Hintergrund der Rolle der Zentralbank innerhalb des Maastrichter Regimes der europäischen WWU und schließt somit auch eine Beurteilung eben dieses Regimes mit ein. In der Tat ist die EZB die zentrale makropolitische Institution der WWU. Ihr steht keine ebenbürtige, für die Wirtschafts- und Finanzpolitik verantwortliche Institution gegenüber, wie dies üblicherweise in Nationalstaaten der Fall wäre. Vielmehr betreiben die Mitgliedsländer der WWU weiterhin ihre nationalen Wirtschafts- und Finanzpolitiken, wobei der sogenannte Stabilitäts- und Wachstumspakt (SWP) zwar ihren nationalen Politikfreiraum asymmetrisch beschränkt - verhindert werden sollen allein exzessive Defizite -, aber keine wirkliche Politikkoordinierung beinhaltet. Die makropolitische Ausrichtung europäischer Finanzpolitik sowie der Beitrag der Wirtschafts- und Finanzpolitik zum europäischen MakroPolicy-Mix bleiben damit dem Zufall überlassen. Anders ausgedrückt: Die konjunkturpolitische Schaltzentrale im Euroland ist per Design von der EZB allein besetzt. Die andere zentrale Institution mit Verantwortlichkeiten für die Volkswirtschaft der EU insgesamt, die Europäische Kommission, ist zwar neben ihrer mikroökonomischen Führungsrolle für den gemeinsamen Binnenmarkt auch an der nach Artikel 120 des Vertrags über die Arbeitsweise der EU für die WWU vorgesehenen Politikkoordinierung beteiligt und somit im Prinzip makropolitisch ebenfalls gefordert. Doch beschränken sich die unter ihrer Beteiligung entwickelten „Allgemeinen Grundzüge der Wirtschaftspolitik" erfahrungsgemäß auf gebetsmühlenartig wiederholte Weisheiten zur unbedingten Einhaltung des SWP sowie zur Marktflexibilisierung durch Strukturreform.

Die makropolitische Vorherrschaft der EZB im Maastrichter Regime ist erwünscht. Sie soll deutschen Sorgen über vermutete finanzpolitische Gefahren für die gemeinsame Währung und ihre Hüterin begegnen. Solidität der öffentlichen Finanzen der Mitgliedsländer soll es der EZB ermöglichen, als unanfechtbarer Garant der Stabilität des Euro zu wirken. Nach dem Vorbild der Deutschen Bundesbank hat die EZB hierzu unabhängig von jeglichem politischen Einfluss zu sein. Tatsächlich ist die EZB die wohl unabhängigste, also die am wenigsten in ihren Handlungsfreiräumen beschränkte Zentralbank der Welt - und dabei keiner politischen Rechenschaftspflicht unterworfen. Zwar unterliegt die EZB einigen Veröffentlichungspflichten zu ihren Aktivitäten, was sie als treffliche Gelegenheit zur Public-Relations-Pflege ausgemacht hat, und ihr Präsident tritt einmal im Quartal vor einen Ausschuss des Europäischen Parlaments, um Aus-

Jörg Bibow, Dr., ist Professor of Economics am Skidmore College, Saratoga Springs, New York, USA und Forscher am Levy Economics Institute of Bard College, Annandale-onHudson, New York. Arbeitsschwerpunkte: Europäische Integration, Monetäre Makroökonomie, Internationale Finanzen, Zentralbankwesen, Finanzmärkte und -institutionen. e-mail: jbibow@skidmore.edu 
kunft zu erteilen. Doch von einer wirksamen Rechenschaft und Aufsicht kann dabei keine Rede sein. Denn es gibt keine Ahndung von Fehlern, keine Sanktionen. Nicht krasse Inkompetenz, sondern allein politische Unmöglichkeit kann den Zentralbankpolitikern der WWU bei aller Unabhängigkeit, die sie genießen, noch zum Verhängnis werden. Ihre Unabhängigkeit hat durch den Maastrichter Vertrag quasi Verfassungsrang erlangt, was den rechtlichen Status der Unantastbarkeit der Währungshüter im Vergleich zum Bundesbankgesetz der Vor-Euro-Zeiten enorm ausbaute.

Die EZB ist also nicht nur zentrale makropolitische Institution der WWU ohne jegliches finanzpolitisches Pendant, sondern auch politisch unanfechtbar. Die Designer des Maastrichter Regimes entsprachen damit deutschen Vorbedingungen für eine WWU, und in Deutschland ist auch 20 Jahre nach dem Maastrichter Vertrag nicht an den, diesem Regime zugrunde liegenden Glaubenssätzen zu rütteln. Eine starke Währung - so das Credo - braucht eine starke Zentralbank. Und eine Zentralbank ist vermeintlich dann am stärksten, wenn ihre potenziellen Gegner kaltgestellt sind. Überhaupt gilt es ja nicht, die Wirtschaft durch gezielte Makropolitik zu stabilisieren, sondern die Politik selbst auf Stabilität festzulegen. Der angelsächsischen Konzeption von Stabilisierungspolitik wird das deutsche Konzept der Stabilitätspolitik entgegengesetzt. Nicht Konjunkturpolitik, sondern „stabilitätsorientierte“ Geldpolitik ist der Angelpunkt der WWU. Wie die EZB diesen Anspruch stabilitätsorientierter Geldpolitik mit Leben zu erfüllen versucht hat, wird im nächsten Abschnitt behandelt. Zuvor gilt es, kurz einige der deutschen Glaubenssätze etwas kritischer zu beleuchten, die das Maastrichter Regime inspirierten.

Zum Beispiel ist eine unanfechtbare und auf sich allein gestellte Zentralbank nicht zwangsläufig stark, sondern findet sich in kritischen Situationen vielleicht ohne Partner und politischen Rückhalt wieder. Das britische Schatzamt ist nicht zwingend gefährlicher Gegenspieler der Bank of England oder das amerikanische Schatzamt ein solcher der US Federal Reserve, sondern sie sind wichtige Partner in der Bewältigung wirtschaftspolitischer Herausforderungen. Dass diese beiden Zentralbanken einer gewissen politischen Kontrolle durch ihre jeweiligen nationalen
Parlamente unterliegen, bedeutet in der Praxis auch den Genuss politischen Rückhalts durch die Träger letzter politischer Gewalt im Staate. Vielleicht mögen die Designer des Maastrichter Regimes von einer Hayekschen „Entnationalisierung des Geldes" geträumt haben, als sie den Euro als Währung ohne Staat, als Währungsunion ohne Politische Union schufen. Aber so ist die EZB eben auch eine Zentralbank ohne Schatzamt oder Wirtschaftsregierung als Partner und ohne Rückendeckung, was sich speziell währungspolitisch sowie in Finanzkrisen als Schwachpunkt statt Stärke der Zentralbank - und damit der WWU - erweisen kann. Fraglich ist auch, ob Geldpolitik durch eine übertriebene Unabhängigkeit der Zentralbank wirksam „entpolitisiert" wird, oder ob nicht vielmehr die Zentralbank als politischer Akteur positioniert wird, der außerhalb der üblichen demokratischen „checks and balances“ agiert. Ähnlich wie zuvor die Deutsche Bundesbank verweist auch die EZB auf ihre vermeintliche direkte Rechenschaftspflicht gegenüber der Öffentlichkeit anstelle politischer Organe. Genau hierin konzentriert sich allerdings die Gefahr, dass sich Zentralbankpolitiker zur Steigerung des eigenen Ansehens als Gegenspieler der für die Wirtschaftsund Finanzpolitik verantwortlichen, demokratisch gewählten Politiker gerieren; negative Folgen für die Politikkoordinierung macht dies umso wahrscheinlicher.

Auf den Punkt gebracht hat der Maastrichter Vertrag eine WWU ohne konjunkturpolitische Steuerung der Binnennachfrage geschaffen, sofern nicht die auch in dieser Entscheidung uneingeschränkt unabhängige EZB diese Verantwortung freiwillig auf sich nimmt - einmal unterstellt, dass eine Zentralbank diese Aufgabe überhaupt allein erfüllen kann. Konjunkturpolitische Abstinenz entspricht dem Vorbild deutscher Stabilitätspolitik. Es darf gefragt werden, ob eine große, offene Volkswirtschaft Stabilität und Wachstum erreichen kann, wenn mikropolitisch allein auf Marktflexibilität und makropolitisch allein auf „stabilitätsorientierte“ Geldpolitik gesetzt wird. Wie ich im nächsten Abschnitt darstelle, geben die Erfahrungen der WWU hinreichenden Grund zur Ernüchterung.

\section{Gelebte deutsche Stabili- tätskultur mit Eigentoren am laufenden Band}

\subsection{DIE ASYMMETRISCHEN INTERVENTIONEN DER EZB}

Das für die Wirtschaftsentwicklung im Euroland entscheidende Markenzeichen der EZB ist die ausgeprägte Asymmetrie in ihrer Geisteshaltung und praktischen Geldpolitik. Das Mandat der EZB reserviert zwar eine Vorrangstellung für das Ziel der Preisstabilität, überlässt es aber den unabhängigen Zentralbankpolitikern, ihren Auftrag zu interpretieren. Einen klaren Hinweis für ihre asymmetrische Haltung lieferte bereits die von der EZB selbst gewählte Definition von Preisstabilität als eine Inflationsrate von „unter zwei Prozent“ (EZB 1999). Da der Mangel an expliziter Symmetrie selbst von Mainstream-Ökonomen scharf kritisiert worden war, erfolgte mit der Revision ihrer Strategie im Mai 2003 eine „Klarstellung“ der Definition von Preisstabilität als Inflationsrate von „unterhalb aber nahe zwei Prozent" (EZB 2003). Welche Bedeutung andere Ziele neben der Wahrung von Preisstabilität haben, wie etwa Wachstum und Beschäftigung - sekundäre Ziele, deren Verfolgung die EZB zu unterstützen hat, sofern dies nicht ihr Preisstabilitätsziel gefährdet -, machte sie dagegen von Beginn an klar: „maintaining price stability in itself contributes to the achievement of output and employment goals“ (EZB 1999, Hervorhebung des Autors). Preisstabilität explizit als „einziges“ statt „vorrangiges“ Ziel der EZB beschreibend, erklärte Wim Duisenberg hierzu dem Währungsausschuss des Europäischen Parlaments: „we have always maintained - and we still do - that the best contribution that monetary policy can give to fulfill that second task is to maintain price stability" (Duisenberg 2001). Und ähnlich bemühte Jean-Claude Trichet die Metapher vom EZB Kompass, der nur eine Nadel hätte. Die EZB will keine Konjunkturpolitik betreiben. In ihrem geldpolitischen Verständnis ist jeglicher „Aktivismus“ oder „fine tuning“ strikt abzulehnen. Erzeugen könne dies bestenfalls sogenannte Strohfeuer und schlimmstenfalls Inflation (Issing 2003). Stattdessen gibt sie vor, eine „Politik der 
ruhigen Hand" zu betreiben, die sich allein auf die Wahrung der Preisstabilität in der mittleren Frist konzentriert.

Tatsache ist dagegen, dass die EZB entsprechend ihrer Geisteshaltung auch in der Zinspolitik ausgesprochen asymmetrisch agiert: Sie legt bei konjunkturell angezeigter Lockerung ein gemächliches Tempo vor, reagiert erst nach langer Verzögerung, scheut sich dagegen nicht vor übereilter Vollbremsung, sobald sie an irgendeinem Horizont Inflationsrisiken auszumachen glaubt (Abbildung 1). So lassen sich seit Beginn der WWU bislang zwei Zinszyklen ausmachen. Nach Jahren der Disinflation und Konsolidierung im Zeichen von Maastricht sowie vor dem Hintergrund der Asien- und Russlandkrisen startete die WWU bei hoher Unsicherheit und niedrigem Zinsniveau. Als sich die Weltkonjunktur dann aufhellte und der lange US-Aufschwung der 1990er Jahre zum Endspurt ansetzte, straffte die EZB rasant ihren Kurs, verdoppelte ihren Leitzins bis Oktober 2000 fast. Dies trug zum Euroabsturz und dadurch bedingten Inflationsanstieg in den ersten Jahren der gemeinsamen Währung gehörig bei, da die EZB aus den Augen verloren hatte, dass sich die Finanzmärkte durchaus für Wachstum interessieren und daher eine als wachstumsfeindlich eingeschätzte Geldpolitik abstrafen mögen (Bibow 2004). Ganz anders reagierte die EZB dann auf den globalen Konjunkturabschwung 2001. Nur mit extremer Verzögerung senkte sie den Zins bis Juni 2003 auf $2 \%$. Die Binnennachfrage, insbesondere in Deutschland, blieb für Jahre in hartnäckiger Stagnation stecken. Der Euro wertete dagegen ab 2002 stark auf, was von amerikanischer Seite aktiv betrieben wurde, weil man dort Deflationsrisiken und Arbeitslosigkeit mit allen Mitteln zu begegnen suchte. Andere Länder wie etwa China und Japan entgegneten den Aufwertungstendenzen ihrer Währungen u.a. mit Devisenmarktinterventionen. Trichet versuchte es zwar mit verbalen Interventionen, doch insgesamt zeigte sich die WWU auch in dieser Phase durch das Fehlen einer Währungspolitik verwundbar. Entspannung brachte erst das Anspringen der amerikanischen Konjunktur und die dadurch bedingte geldpolitische Straffung seitens der Federal Reserve im Frühjahr 2004. Im Ergebnis musste die WWU lange auf das Überspringen der boomenden Weltkonjunktur warten. Trotzdem trat die EZB Ende 2005 erneut

\section{Abb. 1: Binnennachfrage und Leitzins im Euroraum}

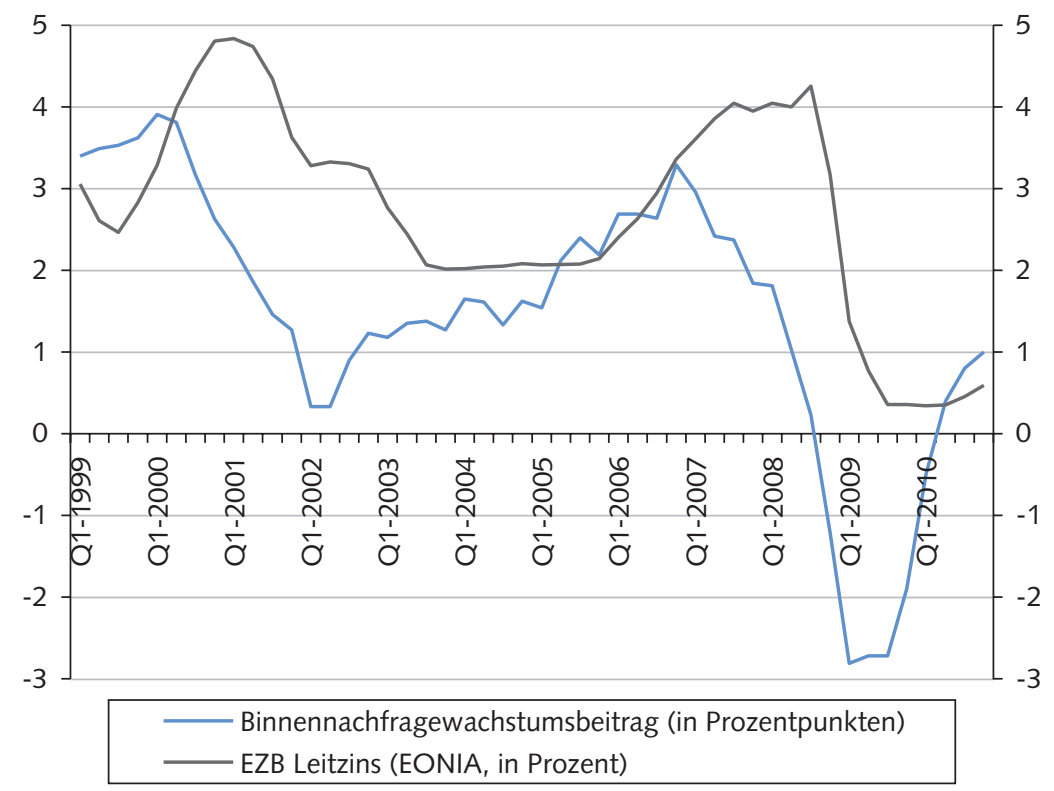

Anmerkung: Wachstumsbeitrag der Binnennachfrage (exkl. Lagerveränderung).

Quelle: Eurostat, EZB.

WSI MitTEILUNGEN

scharf auf die Bremse. Zwar verhielt sie sich im Vergleich zur ersten Vollbremsung im Jahr 2000 etwas vorsichtiger, verschlief aber dafür die Konjunkturabkühlung ab 2007 umso deutlicher: Noch im Juli 2008, also ein Jahr nach Beginn der Verwerfungen auf den globalen Finanzmärkten, erhöhte sie ihren Leitzins. Erst nach der Lehman-Krise trat sie den Kurswechsel an. Vor diesem Hintergrund verwundert es nicht, dass der Konjunkturverlauf im Euroland durch lange Phasen schwachen Binnennachfragewachstums gekennzeichnet ist, die durch nur kurze Phasen des Booms unterbrochen werden, wenn sich weltwirtschaftliche Impulse als hinreichend stark erweisen sollten. Das Bild für Deutschland ist dabei besonders krass: Die deutsche Konjunktur wird fast ausschließlich vom Export getragen. Im Gegensatz dazu ist die US-Konjunktur durch lange Phasen des Booms gekennzeichnet, die durch eher kurze Rezessionen unterbrochen werden, auf die makropolitisch schlagkräftig reagiert wird. Aber die EZB will eben keine Konjunkturpolitik betreiben, jedenfalls nicht, wenn es um die Anregung der Konjunktur geht. Beim gezielten Abwürgen zeigt sie dagegen niemals Hemmungen. Wie in Abschnitt 2 beschrieben, ist das Problem der WWU, dass niemand für die Konjunktur zuständig ist, sofern sich nicht die EZB freiwillig dazu bekennen sollte.
Die asymmetrische Geldpolitik der EZB bewirkt allerdings genau das Gegenteil des Erforderlichen. Im Zusammenwirken mit der Fiskalpolitik ergeben sich aus diesem Versagen gravierende Konsequenzen für die WWU.

\subsection{KERNINFLATION UND STEUEREFFEKTE}

Der Autor hat noch vor Beginn der WWU auf die Gefahr verwiesen, dass sich der sogenannte SWP als „Instabilitäts- und Stagnationspakt" (Bibow 2001) entpuppen würde, sofern nicht die EZB für hinreichend starkes, von der Binnennachfrage getragenes Wachstum Sorge trage. Die Folgen der asymmetrischen EZB-Politik waren in den Jahren 2002 bis 2005 wirklich verheerend und selbst im Hinblick auf das ,einzige“ $\mathrm{Po}$ litikziel der EZB äußerst kontraproduktiv. Stagnationsbedingt gerieten nämlich infolge des Abschwungs von 2001 die meisten WWU-Länder in Konflikt mit dem SWP. Ihre Versuche, die Budgetlage bei Stagnation zu verbessern, enthielten viele Erhöhungen von administrierten Preisen und indirekten Steuern. Diese wirken allerdings preistreibend, halten also die gemessene Inflation künstlich hoch, obgleich disinflationäre Marktkräfte an sich eine geldpolitische Lockerung induzieren sollten. Sie würden dies jedenfalls dann, wenn die Geldpolitik 
ihr Augenmerk auf Marktsignale richtete, die etwa in Form einer Kerninflationsrate messbar sind, welche die „Headline“-Inflation nicht nur um volatile Energie- und Nahrungsmittelpreise (s. Kerninflation 1), sondern auch um etwaige Steuereffekte („Tax-Push“) bereinigt (s. Kerninflation 2). Abbildung 2 zeigt den beträchtlichen Inflationsbeitrag von „Tax-Push“(Bibow 2006a) in dieser Phase .

Sie zeigt auch, dass die marktbedingte Inflationsrate in fast der gesamten Zeit unterhalb von $2 \%$ blieb. Für die EZB waren ihre beständigen Zielverfehlungen dagegen Grund, geldpolitische Lockerungen zu verschleppen. Im Ergebnis hat sie damit jedoch ihren eigenen Zielen geschadet. Denn ihre konjunkturpolitische Verweigerungshaltung erzeugte genau jene Budgetzwänge, die für ihre eigenen Zielverfehlungen im maßgeblichen Umfang ursächlich waren. Die Designfehler des Maastrichter Regimes lassen sich kaum besser veranschaulichen. Die nationalen Finanzpolitiken sind in beeindruckender Einfältigkeit asymmetrisch auf die magische $3 \%$-Defizitmarke vergattert. Und auch die asymmetrische Zentralbank hat sich in fürstlicher Genügsamkeit engstirnig auf die magische $2 \%$-Preisstabilitätsnorm verrannt. Koor- dinierung und Kooperation finden nicht statt; konjunkturpolitische Partnerschaft und Teamgeist sind nicht gefragt. Man legt sich lieber gegenseitig lahm, und verhagelt dem Gegenspieler damit die Zeugnisnote, die gleichsam für die gewählten Finanzpolitiker wie für unabhängige Zentralbankpolitiker „mangelhaft“ ausfällt.

\subsection{LEISTUNGSBILANZEN UND DIVERGIERENDE LOHNSTÜCK- KOSTEN}

Doch hier hört der durch Fehlkonstruktion des Maastrichter Regimes in der WWU verursachte volkswirtschaftliche Schaden noch lange nicht auf. Denn in der anhaltenden konjunkturellen Schwächephase nach 2001 wurden auch jene Fehlentwicklungen maßgeblich verstärkt, die sich dann in der Folgezeit zuerst in der globalen Finanzkrise und wenig später in der sogenannten europäischen Staatsschuldenkrise entladen sollten. Hintergrund der heutigen Krise der WWU sind jene regionalen Leistungsbilanzungleichgewichte innerhalb der WWU, die sich - durch das Maastrichter Regime selbstverstärkend - über Wachstumsdivergenzen und (Quasi-)Wechselkursverschiebungen bis zur Krise immer weiter auftürmten. Die Kernursache hierfür ist in divergierenden Lohnstückkostentrends zu sehen, wie sie seit Ende der 1990er Jahre und verstärkt nach 2001 innerhalb der WWU auftraten.

Vor dem Hintergrund der Erfahrungen der Niederlande in den 1990er Jahren hatte der Autor auch diese Gefahr früh gesehen (Bibow 2001). Die Niederlande hatten sich damals auf dem Wege der relativen Lohndeflation eine Quasi-Abwertung erschlichen und so über den Export bei aus dem Nachbarland Deutschland importierter Geldpolitik ein viel gepriesenes Beschäftigungswunder erzielt. Im Falle einer kleinen und sehr offenen Volkswirtschaft funktionierte diese Strategie reibungslos und ohne eine Binnennachfrageschwäche $\mathrm{zu}$ verursachen. Ich warnte, dass diese Strategie weder für größere Länder noch gar für Euroland als Ganzes eine Option wäre. Vielmehr komme es innerhalb der WWU zu folgeschweren Verwerfungen, da divergierende Lohntrends (produktivitätsbereinigt) als Quasi-Wechselkurse zu Verzerrungen der relativen Wettbewerbsposition führten, und zwar mit kumulativen Wirkungen.

Nach der Euroeinführung wurde Deutschland selbst zur treibenden zer-

\section{Abb. 2: Steuereffekte (Tax-Push) und Inflation im Euroraum}

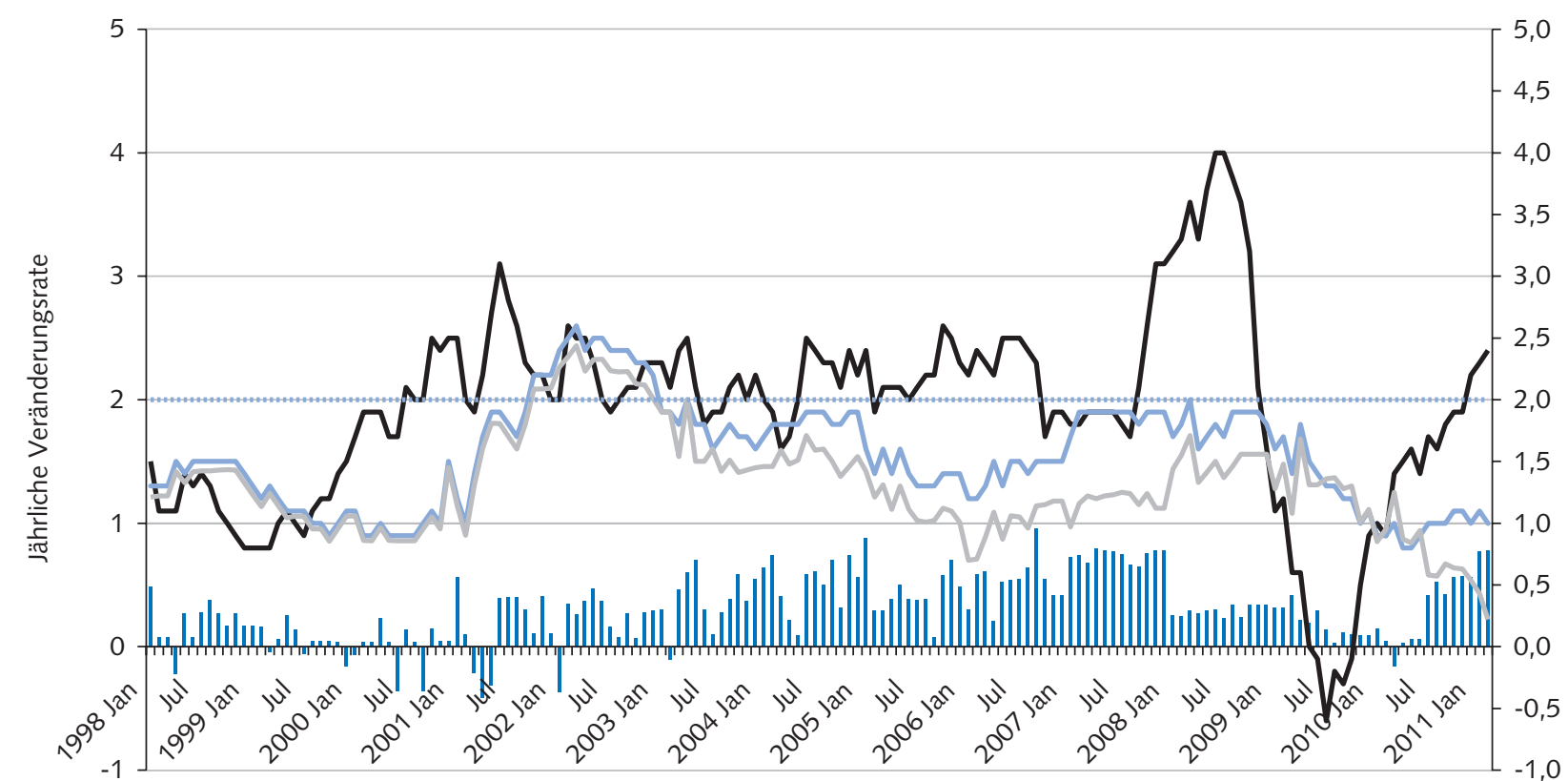

"Tax Push" (rechte Skala) _ Headline Kerninflation $1 \quad$ Kerninflation 2


störerischen Kraft innerhalb der europäischen Partnerschaft. Abwertungen zur Verbesserung der Wettbewerbsposition („beggar-thy-neighbour“) waren aus der währungspolitischen Trickkiste kontraproduktiver Politiken verbannt. Also begann Deutschland noch zu Zeiten der alten D-Mark, die katastrophalen Folgen verfehlter Makropolitik in Reaktion auf die historische Herausforderung „Wiedervereinigung“ mit einem „Bündnis für Arbeit“, einem Bündnis zur Lohndrückung, zu bearbeiten. Es sollte nach dem alten, in den 1990er Jahren in den Niederlanden erprobten Model „Exportmotor“ funktionieren. Vor Maastricht genügte es dazu, auf die höhere Inflation in den Handelspartnerländern zu vertrauen. Nunmehr waren diese auf das niedrige deutsche Niveau konvergiert - wie es ja in der Maastrichter Stabilitätsgemeinschaft nach deutscher Prägung zu geschehen hatte. So schaltete man in Deutschland auf einen noch niedrigeren Gang, die Löhne stiegen bestenfalls noch mit der Inflationsrate, die Arbeitnehmer wurden vom Produktivitätsfortschritt abgekoppelt. Die von entsprechenden Interessen viel gepriesenen Hartz-Reformen der Schröder-Regierung taten ihr Übriges. Gewinneinkommensbezieher und Aktionäre hatten ihre wahre Freude. Zu Ende gedacht hatte man das allerdings nicht.

Auf zahlreichen europäischen Konferenzen, u.a. einer Brüsseler Tagung, auf der auch Vertreter der entscheidenden europäischen Institutionen anwesend waren, warnte der Autor davor, dass die durch divergierende Lohntrends verursachten Leistungsbilanzungleichgewichte in einer Krise enden würden (z. B. Bibow 2006b, 2006c). Dem wurde entgegnet, dass solche Ungleichgewichte innerhalb einer Währungsunion nicht mehr von Belang seien; automatische Marktkräfte würden über den „Wettbewerbskanal“ stabilisierend wirken und das Gleichgewicht innerhalb der Union wiederherstellen (bzw. bewahren). Strukturreformen zur Erhöhung der Marktflexibilität würden die Anpassungsfähigkeit des Systems weiter verstärken.

Übersehen wurde hier, dass das Maastrichter Regime den durch ungleichgewichtige Lohntrends losgetretenen Divergenzen nicht entgegenwirkt, sondern diese sogar verstärkt, jedenfalls bis es irgendwann zu echten Turbulenzen kommt. In Deutschland etwa bewirkte Lohnzurückhaltung über eine relativ niedrige Inflationsrate im Vergleich zu den Partnern hohe Realzin- sen. In Kombination mit stagnierenden Arbeitnehmereinkommen belastete dies die Konsumnachfrage und Hauspreisentwicklung. Auch der SWP verstärkte die deutsche Binnennachfrageschwäche, etwa über Kürzungen der öffentlichen Investitionen und immer neue Beschneidungen der verfügbaren Einkommen. Dies wirkte über den Tax-Push-Effekt fiskalischer Maßnahmen im Tandem mit der EZB gleich doppelt destabilisierend.

Ganz anders war etwa die Situation in Spanien. Dort hatte der Mitte der neunziger Jahre einsetzende Zinskonvergenzprozess dem spanischen Immobilienmarkt und der Binnennachfrage kräftig Anschub gegeben. Über hohes Beschäftigungswachstum bei steigenden Löhnen wurde hieraus ein langer, selbsttragender Aufschwung genährt. Entsprechend entwickelten sich die spanischen Lohnstückkosten sowie die Konsumpreisinflation stärker als im WWU-Durchschnitt. Das von der EZB gesetzte, für die WWU einheitliche Nominalzinsniveau bedeutete also für Spanien deutlich niedrigere Realzinsen als etwa für Deutschland. Der spanische Immobilienmarkt erhielt so weiteren Anschub. Aufgrund des langen Aufschwungs stand es auch um die öffentlichen Finanzen Spaniens ausgezeichnet. Die Schuldenstandsquote war kräftig auf unter $40 \%$ gesunken. Der Staatshaushalt war in den Jahren vor 2008 ausgeglichen oder sogar im Überschuss. Vom SWP ging also kein Konsolidierungsdruck aus, denn der Pakt diszipliniert asymmetrisch und wirkt im Ergebnis prozyklisch, Divergenzen innerhalb der WWU verstärkend: Das stagnierende Deutschland wird zu Steuererhöhungen verdammt, das florierende Spanien kann sogar Steuern senken. Diese sich über das Maastrichter Regime selbstverstärkenden Prozesse dauern an, solange ihre Finanzierung gewährleistet ist.

In der Tat wurden die aufgrund dieser Divergenzen innerhalb der WWU immer weiter anschwellenden Leistungsbilanzungleichgewichte bis zum Ausbruch der globalen Krise problemlos durch das immer enger integrierte europäische Finanzsystem finanziert. Insbesondere deutsche Banken haben sich dabei hervorgetan, was wenig verwunderlich ist. Denn ein Land, das permanente Handelsüberschüsse einfährt, muss zwangsläufig seine Vermögensposition gegenüber dem Ausland entsprechend ausbauen. Abgesehen von der Finanzierung des Exportgeschäfts ihrer deutschen Industriekunden sowie etwaigen Engage- ments im Kreditgeschäft mit Nichtbanken in den deutlich stärker als in Deutschland wachsenden Handelsbilanzdefizitländern wachsen die Forderungspositionen deutscher Banken im Interbankengeschäft mit den Bankensystemen der Defizitländer. Hinzu kommen entsprechende Positionen über die Wertpapiermärkte, speziell Staatsschuldpapiere. Das Risiko solcher Engagements wurde als gemeinhin niedrig eingeschätzt, da die zugrunde liegenden makroökonomischen Ungleichgewichte nach offizieller Meinung gutmütiger Natur waren. Mikroökonomisch wurde diese Kreditrisikoeinschätzung untermauert durch starke Ratings, die wiederum durch die positive Entwicklung der Kreditsicherheiten bzw. der günstigen Haushaltslage öffentlicher Schuldner gestützt schienen.

Anders als die zuvor behandelten Kritikpunkte erlauben diese zuletzt behandelten Fehlentwicklungen keine direkte Kritik an der Politik der EZB. Die EZB kann bestenfalls eine Geldpolitik betreiben, die für die WWU insgesamt geeignet ist, also den Erfordernissen der Länder und Regionen im Durchschnitt gerecht wird. Wie von der Theorie optimaler Währungsräume herausgearbeitet wurde, untergraben Divergenzen innerhalb des Währungsraumes die einheitliche Geldpolitik. Lohnzurückhaltung in Deutschland war eben keine angemessene-stabilisierende-Reaktion auf einen asymmetrischen Schock. Vielmehr war das deutsche Lohndumping selbst der asymmetrische Schock, der die EZB-Politik untergraben und die WWU destabilisieren würde.

Der EZB ist jedoch auch in dieser Hinsicht zumindest mittelbar schwerstes Versagen vorzuwerfen. Denn die im Wesentlichen durch Deutschlands Lohndeflation bedingten Divergenzen und Ungleichgewichte wurden von der EZB nicht nur gründlich fehlinterpretiert und die sich darin andeutende zwangsläufige Krise, mit der Europa heute zu kämpfen hat, verschlafen, sondern vielmehr als Musterbeispiel stabilitätsorientierten Verhaltens gepriesen. Nicht Deutschland hat sich nach dieser Sichtweise fehlverhalten, indem es immer größere Handelsüberschüsse einfuhr. Sünder sind allein die Defizitländer. Diese absurde Beurteilung macht dann allerdings auch Frankreich zum „Sünder“, wo die Löhne sich zwar entsprechend der impliziten „Eurostabilitätsnorm“ (Produktivitätswachstum plus EZB-Preisstabilitätsnorm) entwickelten und das stabile 
Wirtschaftswachstum durch die Binnennachfrage getragen wurde, dabei allerdings deutliche Wettbewerbsverluste gegenüber Deutschland erduldet wurden. Dies unterstreicht einmal mehr den pathologischen Befund einer ausgeprägten Asymmetrie: Für die EZB können Lohnänderungsraten gar nicht zu niedrig sein, selbst eine stabilitätsgerechte Lohnpolitik kann dagegen Sünde sein. Es verwundert nicht, dass eine fehlkonzipierte WWU und die von der zentralen makroökonomischen Institution dieser WWU zu verantwortende irregeleitete Geldpolitik Europa in eine katastrophale Krise führten.

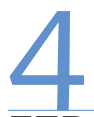

\section{EZB und WWU in der globalen Finanzkrise}

\subsection{IM ZENTRUM DER KRISE ODER KAUM BETROFFEN?}

Die Wirtschaftspolitik der WWU brauchte sehr lange, um überhaupt zu erkennen, dass Europa sich im Zentrum einer globalen Finanzkrise befand. Der ehemalige deutsche Finanzminister Peer Steinbrück kommentierte amerikanische Notmaßnahmen in Reaktion auf den Lehman-Bankrott flott mit den Worten: „this crisis originated in the United States and is mainly hitting the United States ... [In Europe and Germany, such a package would be] neither sensible nor necessary" (zitiert in Benoit 2008). Steinbrück drückte darin auch die von der EZB geteilte Meinung aus, dass die Ursachen für die globale Krise allein anderswo in der Weltwirtschaft lägen, mit der WWU aber nichts zu tun hätten. Auch in den Jahren zuvor hatte man jegliche Verantwortung für die viel diskutierten globalen Ungleichgewichte abgestritten und die im letzten Abschnitt besprochenen regionalen Ungleichgewichte dabei schön gedichtet. Die EZB hatte tatsächlich noch im Juli - in typischer Inflationspanik - ihren Zins gestrafft, sie wurde erst durch die Lehman-Krise wachgerüttelt.

Tatsächlich war Europa von Beginn an im Zentrum der dem Anschein nach allein von den USA ausgehenden Krise, die zur schwersten Finanz- und Weltwirtschaftskrise seit den 1930er Jahren wurde. Die Fehlkonstruktion des Maastrichter Regimes sowie Versagen der EZB waren maßgeblich dafür verantwortlich, dass die Krise zu einer schweren globalen Krise wurde, sich die WWU 2010 global betrachtet am schwächsten von allen Regionen erholte und heute auf den Märkten unter der Bezeichnung "Staatsschuldenkrise“ als Wackelkandidat der Weltkonjunktur gehandelt wird. Die WWU hat nicht nur eine große offene Volkswirtschaft geschaffen, die auf Grund fehlender konjunkturpolitischer Steuerung bzw. Verteidigung extrem verwundbar gegenüber weltwirtschaftlichen Schocks ist. Die WWU basiert auch auf einem gemeinsamen Markt, der zwar tiefe Finanzintegration beinhaltet, aber keine wirkliche gemeinsame Finanzmarktaufsicht und Finanzkrisenpolitik. Gemeinsame Finanzkrisenpolitik fehlt, weil die WWU keine Fiskalunion ist. Etwaige Rettungsaktionen, etwa von insolventen Banken, müssen also aus nationalen Budgets erfolgen. Allerdings können sowohl nationale Bankenkrisen als auch Rettungsaktionen Spillover-Effekte innerhalb der WWU (und darüber hinaus) haben, also den gemeinsamen Markt und die WWU untergraben.

In den 1980er Jahren war die Europäische Gemeinschaft zur treibenden Kraft von Finanzliberalisierung und globaler Finanzintegration geworden (Abdelal 2007). Regionale Finanzintegration durch den gemeinsamen Markt machte Europa hierin zum globalen Vorreiter. Die WWU hat dies regional noch weiter verstärkt. Ohne einer wirksamen Kontrolle zu unterliegen, hatten sich Europas Banken freimütig immer stärker jenseits nationaler Grenzen engagiert. Banken aus WWU-Ländern hatten sich stark gegenüber Kreditrisiken aus dem amerikanischen Immobilienmarkt exponiert. Und sie hatten sich auch stark in den neuen EU-Mitgliedsländern engagiert. Aufgrund der im letzten Abschnitt behandelten inneren Ungleichgewichte waren die Bankensysteme der WWU-Mitgliedsländer nicht nur eng untereinander vernetzt, sondern es hatten sich auch große NettoForderungspositionen aufgestaut. Grundsätzlich ergibt regionale Finanzintegration innerhalb einer WWU ökonomisch Sinn, da die gemeinsame Geldpolitik innerhalb des Währungsraumes in Form weitgehend einheitlicher Finanzbedingungen Entfaltung finden muss, um wirksam und gezielt steuerbar zu sein. Untragbare Ungleichgewichte, wie sie in der WWU entstanden waren, bedingen allerdings Fragilität und gegenseitige Verwundbarkeit. Entladen sich diese Ungleichgewichte und führen dabei zu systemischen Liquiditätsengpässen, ist die gemeinsame Zentralbank als „Bereitsteller letzter Liquidität“ gefordert. Kommt es dagegen unter europäisch aktiven Banken zu Insolvenzen, die das integrierte Finanzsystem bedrohen, ist die Finanzpolitik gefordert, die allerdings national ist.

\subsection{DIE KRISENREAKTION}

Am 9. August 2007 eskalierten die Verwerfungen auf für Europas Banken zentral wichtigen Finanzmärkten und in der Folgezeit kam es zeitweise zum völligen Erliegen von Interbankengeschäften, speziell am Geldmarkt in längeren Laufzeiten. Die erhöhte Liquiditätspräferenz der Banken und Nichtbereitschaft zu Kreditgeschäften untereinander zeigte sich in steigenden Geldmarktzinsen und der Haltung von Überschussreserven durch individuelle Banken. Gemäß ihrem geldpolitischen Umsetzungsverfahren, das den Banken jene Menge an Zentralbankgeld zur Verfügung stellt, das zum Erreichen des von der EZB angestrebten Tagesgeldzinses (EONIA, euro overnight index average) benötigt wird, reagiert die EZB quasi automatisch auf solche Marktanspannungen, indem sie dem Markt zusätzliche Liquidität zuführt. Dies erfolgte zunächst durch „fine tuning“-Aktionen vermehrter Übernacht-Liquidität. In der Folgezeit hat die EZB ihr Verfahren verfeinert und den Umfang zusätzlich bereitgestellter Liquidität erhöht - eine Politik, die sie dann als „enhanced credit support" bezeichnete. So stellte sie den Banken ab Oktober 2008 „unbegrenzte“ Zentralbankliquidität zur Verfügung, setzte verstärkt Geschäfte mit längeren Laufzeiten (von bis zu zwölf Monaten) ein, weitete die Liste akzeptabler Kreditsicherheiten aus und legte im Mai 2009 sogar ein spezielles Programm (von allerdings nur $60 \mathrm{Mrd}$. €) zur Wiederbelebung des Pfandbriefmarktes auf. Sie begründete all diese Maßnahmen damit, dass sie dem Funktionieren des geldpolitischen Transmissionsmechanismus und damit der Wirksamkeit der Geldpolitik dienen würden. Dank der Hilfe der amerikanischen Zentralbank konnte die EZB dabei auch mittels Devisenswap-Arrangements von dieser beschaffte Dollarliquidität an Banken in der WWU weiterleiten. Weil Europas Banken ihre Geschäftsexpansion in den Dollarraum kurzfristig finanziert hatten, standen sie im Zentrum der globalen 
Dollarlücke (McGuire/Peter 2009). Weil die Banken das Geschäft untereinander verweigerten, wurde ein bedeutender Teil des Geldmarktes von der EZB als Intermediär ersetzt. Ihre Bilanzsumme verdoppelte sich im Zuge dieser Maßnahmen fast.

Von Beginn an betrachtete die EZB diese Liquiditätsnotmaßnahmen als grundsätzlich von ihrer geldpolitischen Ausrichtung unabhängig. Erst am 8. Oktober 2008 senkte sie ihren Leitzins in einer international koordinierten Kampagne um 50 Basispunkte. Bis Mai 2009 reduzierte sie den Zins ihrer Hauptrefinanzierungsgeschäfte in mehreren Schritten um weitere 275 Basispunkte auf $1 \%$. Tatsächlich steuerte sie EONIA sogar noch deutlich darunter und in Nähe des Zinses auf ihre Einlagenfazilität von 25 Basispunkten.

Im Vergleich zu ihrer Trägheit im Abschwung ab 2001 wirken die Zinssenkungen ab Oktober 2008 zunächst rasant. Zu beachten ist allerdings, dass die EZB sehr spät auf den Wirtschaftsabschwung in der WWU reagierte und ihre Politik sogar noch im Juli 2008 zunächst gestrafft hatte. Als sie dann endlich reagierte, befand sich die Wirtschaft der WWU buchstäblich im freien Fall und auf Depressionskurs. In ihrem Tiefpunkt im Sommer 2009 fiel die Headline-Inflationsrate auf Minus 0,6 \%. $\mathrm{Zu}$ dieser Zeit war der freie Fall der Wirtschaft allerdings bereits beendet. International hatten insbesondere die USA und China massive expansive makropolitische Initiativen ergriffen. Selbst in Europa erfuhr die EZB insbesondere durch von der Europäischen Kommission „koordinierte“ nationale Fiskalprogramme Unterstützung, wobei sich Deutschland untypisch durch ein recht großes keynesianisches Konjunkturprogramm für 2009 bis 2010 hervortat. Seitens der EZB erfuhr dies bestenfalls gemischte Billigung: Bereits im Sommer 2009 wurden die Stimmen für eine Rückkehr zur Konsolidierungspolitik und einen Ausstieg aus dem Liquiditätsnothilfeprogramm lauter. Ein solcher Ausstieg wurde dann auch tatsächlich schon Anfang Dezember 2009 initiiert. Der Euro hatte bis dahin deutlich gegenüber dem Dollar aufgewertet. Die EZB war sichtlich bemüht, weniger aggressive Unterstützung als die Federal Reserve zu leisten, deren Bilanzsumme sich inzwischen verdreifacht hatte.

Doch dann kam alles anders. Die sogenannte "Griechenland-Krise" eskalierte und im Frühjahr 2010 einigten sich die
WWU-Partner zunächst auf ein in der Presse als „Griechenland-Rettungspaket“ bezeichnetes Kreditprogramm und dann auf die Europäische Finanzstabilitätsfazilität. Die Krise, die letztlich auf die im Abschnitt 3 beschriebenen Fehlentwicklungen zurückzuführen ist, wurde allerdings bis heute nicht durch wirksame Mittel behoben. Man versucht weiterhin, bestehende mangelnde Wettbewerbsfähigkeit und dadurch bedingte Überschuldungsprobleme von Partnerländern dilettantisch durch Liquiditätshilfen zu verpflastern. Die Liquiditätshilfen werden noch dazu nur unter der Bedingung brutaler Sparmaßnahmen und bei hohen Zinsen gewährt. Nur ist die Schuldendynamik so nicht in den Griff zu bekommen und die Schuldenlast steigt im Ergebnis weiter an. Liquidität kann den Bankrott eben nicht vermeiden, sondern nur herauszögern.

Griechenland ist dabei übrigens ein Sonderfall, weil exzessive Haushaltsdefizite wirklich eine Ursache der Krise waren. In anderen Ländern wie Irland und Spanien war es zunächst die Überschuldung von privaten Haushalten, die über Not leidende Kredite zu Problemen der Solvenz im Bankensystem führten und schließlich durch staatliche Rettungsprogramme der Banken zum Staatsschuldenproblem wurden. Permanente - nur scheinbar tugendhafte - Handelsüberschüsse drängen die WWU Defizitländer in den sicheren Bankrott. So wird die Währungsunion durch die Hintertür zu einer Fiskalunion (Bibow 2010). Diese zwangsläufige Konsequenz deutscher Lohnunterbietungspolitik wird politisch bislang zwar nicht akzeptiert. Währenddessen steigt sowohl in Deutschland als auch in den deflationierenden Partnerländern die Gefahr eines unkontrollierbaren Populismus.

\subsection{EURO UND EUROPÄISCHE INTEGRATION ALS VERLIERER}

So befindet sich auch die EZB inzwischen auf der Verliererbahn. Im Zuge der Rettungsaktionen im Mai 2010 initiierte sie ihr Wertpapiermarktprogramm, erneut begründet als zur Begegnung dysfunktionaler Märkte und zur Gewähr wirksamer Geldpolitik nötig. Im Rahmen dieses Programmes kauft die EZB am Sekundärmarkt Staatsanleihen von WWULändern mit Überschuldungsproblemen (EZB 2010b). Im Januar 2011 erreichten diese Käufe einen Gesamtumfang von
75 Mrd. €. Es wird geschätzt, dass die EZB inzwischen fast $20 \%$ der Staatsschulden von Griechenland, Irland und Portugal hält (Alloway 2011). Zur Beschwichtigung betont die EZB, dass sie keine Titel direkt vom Schuldner übernimmt und ihre Stützungskäufe vollständig sterilisiert, also nicht die Notenpresse zur Staatsschuldenfinanzierung angeworfen hätte. Als kurzfristige Liquiditätshilfen sind solche Stützungskäufe vollkommen erwünscht. Die EZB scheint begriffen zu haben, dass ihr eigenes Schicksal selbst bei unantastbarer Unabhängigkeit am Schicksal des Euro hängt. Sie springt also für den Euro in die Bresche, wenn die Politik nicht handelt. Nur kann auch sie durch die Zwischenlagerung fauler Kredite das eigentliche Problem nicht lösen. Sie fungiert vielmehr als Quasi-Intermediär bei der staatlichen Re-Kapitalisierung der Banken. Statt bei den Banken fallen die Verluste zunächst bei der EZB an, welche dann anstelle der Banken staatlich zu re-kapitalisieren wäre. Die Erhöhung des Eigenkapitals der EZB Ende 2012 macht im Hinblick auf die erneute Ausweitung ihrer Stützungskäufe daher Sinn. Dass ausgerechnet Deutschland bei aller Vergötterung von Zentralbankunabhängigkeit gerade diese Variante bevorzugen soll, unterstreicht nur, in welcher krassen Notlage sich die WWU heute befindet.

\section{Ausblick}

Die Krise hat tatsächlich Anstoß zu Reformen des Maastrichter Regimes der WWU gegeben. So haben Anfang 2011 neu errichtete europäische Aufsichtsbehörden für Banken, Versicherungen und für den Wertpapiersektor ihre Arbeit mit dem Auftrag aufgenommen, harmonisierte Regeln und gemeinsame Herangehensweisen für die Aufsicht zu entwickeln und deren konsistente Anwendung zu sichern. Ergänzend wurde eine neue makroprudenzielle Ebene geschaffen und dazu ein Europäischer Ausschuss für Systemrisiken errichtet. Dieser setzt sich aus den EU-Zentralbankpräsidenten zusammen und wird vom Präsidenten der EZB angeführt. Sein Auftrag lautet, potenzielle Gefahren für die Finanzstabilität in der Region zu beobachten. Dies sind im Prinzip lobenswerte Initiativen zur Vermeidung neuer Finanzkrisen. Ihre Wirksamkeit bleibt abzuwarten. 
Makropolitisch steht eine Verschärfung des SWP im Zentrum der Bemühungen. Ein neu geschaffenes „Europäisches Semester" soll die Umsetzung des Paktes sowie wirksame Politikkoordinierung sicherstellen. Dabei sollen auch gewisse Indikatoren zur Früherkennung von Divergenzen und Ungleichgewichten zum Einsatz kommen. Beschleunigte Durchführung von Strukturreformen soll das Wachstum antreiben, die EZB durch stabilitätsorientierte Geldpolitik weiterhin ihren besten Beitrag für das wirtschaftliche Wohl der WWU erbringen. Kurz gesagt, Europa soll zur Gesundung eine erhöhte Dosis deutscher Stabilitätskultur verabreicht werden. Es bleibt im Ergebnis bei der pathologischen Asymmetrie der Makropolitik, welche-im Maastrichter Vertrag institutionalisiert - Europa in die heutige Krise manövrierte.

Auch bleibt die EZB dabei die Schlüsselinstitution. Ihre Verweigerung symmetrischer Konjunkturpolitik bestimmt die asymmetrische Konjunktur der WWU: lange Phasen schwacher Binnennachfrage, zufällig unterbrochen durch kurze Phasen des Booms, die weltwirtschaftlichen Einflüssen überlassen bleiben. Wir haben im Abschnitt 3 „Tax-Push“ als Indiz der kontraproduktiven Makropolitik der WWU identifiziert. Davon erlebt die WWU heu- te dank brachialer Sparpolitik eine neue Runde. Trotz stabiler und extrem niedriger Kerninflationsrate hat die EZB inzwischen mit der Kursstraffung begonnen. Eine Wiederholung altbekannter Politikfehler scheint sich anzubahnen.

Dies geschieht vor dem Hintergrund der schweren Hypothek jener regionalen Leistungsbilanzungleichgewichte, die sich als Folge divergierender Lohnstückkostentrends herausbildeten und zum EuroSprengsatz und zur Hauptursache der heutigen WWU-Krise wurden. Derartige Divergenzen werden heute zwar nicht mehr als gutmütig erachtet und sollen nunmehr frühzeitig erkannt werden, doch die Schuldzuweisung für derartige Fehlentwicklungen ist höchst interessant. Denn sie unterstreicht das Markenzeichen der EZB: Asymmetrie. So findet der EZB-Präsident lobende Worte für den schmerzhaften Weg, den Deutschland zur Herstellung seiner Wettbewerbsposition durchschritten habe. Zu der naturgemäß spiegelbildlichen Verschlechterung anderer Länder stellt er dagegen fest: „National price and cost developments that are significantly higher than the union average entail significant losses over time in competitiveness. Competitiveness losses cannot be sustained forever, and adjustments in un- sustainable economic policies have to be made. Fiscal and structural policies need to keep domestic demand in line with rates of sustainable growth and price stability" (Trichet 2011, S. 5). Unerwähnt bleibt, dass auch Wettbewerbsgewinne nicht für immer anhalten können, dass Deutschland in der geforderten Sicherung nachhaltigen Binnennachfragewachstums auf krasseste Weise versagt hat, und dass Deutschland allein deshalb seinen Staatshaushalt sanieren konnte, weil die Partnerländer trotz mangelnder Exporte nach Deutschland kräftig aus Deutschland importierten. Bis zum Ausbruch der Krise hat Deutschland die Kredite hierzu mit Fleiß und Begeisterung bereitgestellt. Heute drängt man mit selbiger Begeisterung, die bankrotten Schuldner sollten sich gesund sparen, weil Deutschlands Exporteure nunmehr auf China setzen. Trichets Zuhörer auf der Klausurtagung der CSU-Landesgruppe in Wildbad Kreuth werden die stabilitätsorientierte Lesart des EZB-Präsidenten zwar wohlwollend aufgenommen haben. Sie bedeutet jedoch, dass die Maastrichter WWU mit der EZB als zentraler Makroinstitution nicht nur fehlkonzipiert und die Politik der EZB irregeleitet ist, die EZB erweist sich vielmehr auch im Angesicht der WWUKrise als lernresistent.

\section{LITERATUR}

Abdelal, R. (2007): Capital Rules: The Construction of Global Finance, Cambridge, MA

Alloway, T. (2011): What fresh basis the ECB has wrought - yet again, Financial Times Alphaville Blog, 17. Januar

Benoit, B. (2008): Germany sees an end to U.S. hegemony, FT.com, 26. September

Bibow, J. (2001): Making EMU work: some lessons from the 1990s, in: International Review of Applied Economics 15 (3), S. 233-259

Bibow, J. (2004): Assessing the ECB's performance since the global slowdown - A structural policy bias coming home to roost?, Working Paper 409, hrsg. vom Levy Economics Institute, New York Bibow, J. (2006a): Preistreiber Staat: Zur Inflationspersistenz im Euroraum, IMK Report (6), Januar
Bibow, J. (2006b): The euro area drifting apart - Does reform of labor markets deliver competitive stability or competitive divergence?, in: European Trade Union Confederation (ETUC): Structural Reforms and Macro-Economic Policy, S. 76-86

Bibow, J. (2006c): Europe's quest for monetary stability: central banking gone astray, in: International Journal of Political Economy 35 (1), S. $24-43$

Bibow, J. (2010): Suffocating Europe, Eurointelligence, 25. Juni Duisenberg, W. (2001): Testimony before the Committee on Economic and Monetory Affairs of the European Parliament, 28 May, Brüssel, http://www.ecb.int/press/key/date/2001/html/sp010528.en.html Europäische Zentralbank (EZB) (1999): The stability-oriented monetary policy strategy of the Eurosystem, in: Monatsbericht, Januar, S. 39-50 
Europäische Zentralbank (EZB) (2003): The outcome of the ECB's evaluation of its monetary policy strategy, in: Monatsbericht, Juni, S. 79-92

Europäische Zentralbank (EZB) (2005): Asset price bubbles and monetary policy, in: Monatsbericht, April, S. 47-60

Europäische Zentralbank (EZB) (2009): The implementation of monetary policy since August 2007, Monatsbericht, Juli, S. 75-89

Europäische Zentralbank (EZB) (2009): Recent developments in the balance sheets of the Eurosystem, the Federal Reserve System and the Bank of Japan, in: Monatsbericht, Oktober, S. 81-94

Europäische Zentralbank (EZB) (2010): The ECB's response to the financial crisis, Monatsbericht, Oktober
Issing, O. (2003): Europe and the U.S.: Partners and Competitors. New Paths for the Future, German British Forum, London, 28 October, http://www.ecb.int/key/03/sp031028.htm

McGuire, P./Peter, G. von (2009): The US dollar shortage in global banking and the international policy response, BIS Working Papers (291), Basel

Trichet, J.-C. (2009): The ECB's enhanced credit support, Rede, 13. Juli Trichet, J.-C. (2010): State of the Union: The financial crisis and the ECB's response between 2007 and 2009, in: Journal of Common Market Studies 48 (1), S. 7-19

Trichet, J.-C. (2011): Economic and Monetary Union - what we have achieved and what we must do next, Rede, 7. Januar 